\title{
A FINITE DEFORMATION PHASE-FIELD FRACTURE MODEL FOR NANOPARTICLE/POLYMER COMPOSITES
}

\author{
Behrouz Arash ${ }^{1 *}$ and Raimund Rolfes ${ }^{1}$ \\ ${ }^{1}$ Institute of Structural Analysis, Leibniz University, Appelstraße 9A, 30167 Hannover, Germany \\ *b.arash@isd.uni-hannover.de
}

The computational modeling of fracture in the nanocomposites requires an accurate prediction of crack initiation and propagation in the materials. For this, generalizing Griffith's theory, phase-field fracture models (PFMs) provide variational fracture models by minimizing potential energy that consists of stored bulk energy, the work of external forces, and the surface energy $[1,2]$. This work presents the development of a finite deformation PFM to analyze the viscoelastic behavior of boehmite nanoparticle/epoxy nanocomposites. To characterize the rate-dependent fracture evolution, the free energy is additively decomposed into an equilibrium, a non-equilibrium, and a volumetric part with a varying definition under tensile and compressive deformation. Furthermore, the Guth-Gold and modified Kitagawa models are adopted to consider the effect of the nanoparticle contents and temperature on the nanocomposites' fracture behavior. The applicability of the proposed model is evaluated by comparing the numerical results of compact-tension tests with experimental data. The experimental-numerical validation justifies the predictive capability of the model. Numerical simulations are also performed to study the effect of temperature and loading rate on the force-displacement response of boehmite nanoparticle/epoxy samples in the compacttension tests.
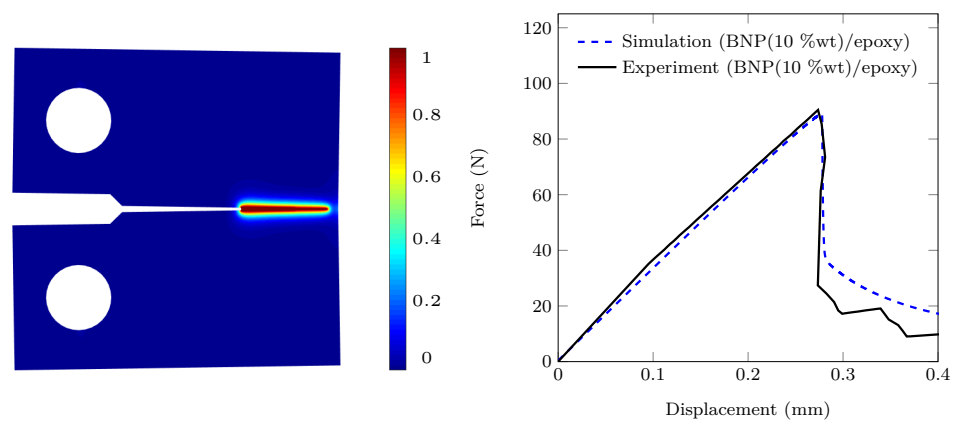

Figure 1: Contour plots of damage for the compact-tension test of a nanoparticle(10\%wt)/epoxy sample (left) and its corresponding force-displacement curve (right).

\section{References}

[1] C. Miehe, F. Welschinger, and M. Hofacker (2010) Thermodynamically consistent phasefield models of fracture: Variational principles and multifield FE implementations. International journal for numerical methods in engineering, 83, 1273-1311.

[2] C. Miehe, M. Hofacker, and F. Welschinger (2010) A phase field model for rateindependent crack propagation: Robust algorithmic implementation based on operator splits. Computer Methods in Applied Mechanics and Engineering, 199, 2765-2778. 